\title{
Alternative lengthening of telomeres: from molecular mechanisms to therapeutic outlooks
}

Jia-Min Zhang ${ }^{1}$ and Lee Zou ${ }^{1,2^{*}}$

\begin{abstract}
To escape replicative senescence, cancer cells have to overcome telomere attrition during DNA replication. Most of cancers rely on telomerase to extend and maintain telomeres, but 4-11\% of cancers use a homologous recombination-based pathway called alternative lengthening of telomeres (ALT). ALT is prevalent in cancers from the mesenchymal origin and usually associates with poor clinical outcome. Given its critical role in protecting telomeres and genomic integrity in tumor cells, ALT is an Achilles heel of tumors and an attractive target for cancer therapy. Here, we review the recent progress in the mechanistic studies of ALT, and discuss the emerging therapeutic strategies to target ALT-positive cancers.
\end{abstract}

Keywords: Telomere, Telomere maintenance mechanism, Alternative lengthening of telomeres (ALT), APBs, Phase separation, ALT telomeric DNA synthesis, RAD52, BLM, FANCM, Clinical therapy

\section{Background}

Telomeres, the ends of chromosomes, are protected by a slew of telomere-binding proteins, which prevent telomeres from being recognized as DNA double-strand breaks (DSBs) $[1,2]$. During the division of somatic cells, the very end of telomeres cannot be replicated by the lagging strand of replication fork, leading to telomere shortening in each cell cycle [3]. If cells continue to proliferate, telomere attrition eventually triggers cellular senescence, a barrier of tumorigenesis [4]. To acquire replicative immortality, cancer cells must overcome telomere shortening [5]. While the majority of cancers accomplish this by activating telomerase [6-8], a significant fraction (4-11\%) uses a telomerase-independent but homologous recombination-based pathway to extend and maintain telomeres [8-10]. This mechanism is known as alternative lengthening of telomeres (ALT) [10-13].

\footnotetext{
*Correspondence: zou.lee@mgh.harvard.edu

${ }^{1}$ Massachusetts General Hospital Cancer Center, Harvard Medical School, Charlestown, MA 02129, USA
}

Full list of author information is available at the end of the article

\section{Hallmarks of ALT}

The telomerase-independent ALT pathway was first identified in the $S$. cerevisiae telomerase mutant, and subsequently characterized in human cancer cell lines and tumors [14-16]. Telomeric DNA synthesis in ALT $^{+}$ cells involves both intra- and inter-telomeric recombination and replication $[17,18]$. $\mathrm{ALT}^{+}$cells display several hallmarks at telomeres, including (1) ALT-associated PML bodies (APBs) [19], (2) heterogeneous telomere length [15], (3) abundant extrachromosomal telomere repeat (ECTR) [20, 21], and (4) high levels of telomere sister chromatid exchange (T-SCE) [22]. Recent studies also found variants of telomeric repeats at telomeres and insertions of telomeric repeats across the genome in $\mathrm{ALT}^{+}$cells $[23,24]$. Using these ALT markers, a spectrum of $\mathrm{ALT}^{+}$cancers are identified, revealing an association of ALT activation with cancers originated from the mesenchymal origin $[9,10,25]$. Sequencing of cancer genomes discovered that mutations of the ATRX/DAXX complex and the histone variant $\mathrm{H} 3.3$ are prevalent in $\mathrm{ALT}^{+}$cancers [26-31]. Although significant progress has been made in phenotypic and sequencing analyses

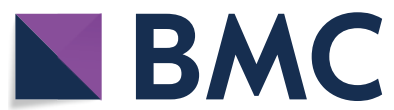

(c) The Author(s) 2020. This article is licensed under a Creative Commons Attribution 4.0 International License, which permits use, sharing, adaptation, distribution and reproduction in any medium or format, as long as you give appropriate credit to the original author(s) and the source, provide a link to the Creative Commons licence, and indicate if changes were made. The images or other third party material in this article are included in the article's Creative Commons licence, unless indicated otherwise in a credit line to the material. If material is not included in the article's Creative Commons licence and your intended use is not permitted by statutory regulation or exceeds the permitted use, you will need to obtain permission directly from the copyright holder. To view a copy of this licence, visit http://creativeco mmons.org/licenses/by/4.0/. The Creative Commons Public Domain Dedication waiver (http://creativecommons.org/publicdomain/ zero/1.0/) applies to the data made available in this article, unless otherwise stated in a credit line to the data. 
of $\mathrm{ALT}^{+}$cells and cancers, ALT is still poorly understood as a pathway at the molecular level. The recent development of assays for telomeric DNA synthesis in $\mathrm{ALT}^{+}$cells has finally allowed us to gain insights into the molecular mechanism of ALT. Here, we will review the recent findings on the molecular mechanisms of the ALT pathway and discuss the potential strategies to target the ALT pathway in cancer therapy.

\section{APBs and spatial regulation of ALT}

APBs are unique nuclear structures containing the promyelocytic leukemia (PML) protein and telomeric DNA, and they are specifically present in $\mathrm{ALT}^{+}$cells [19]. APBs typically contain clusters of multiple telomeres and numerous proteins involved in DNA repair, recombination and replication, making them an ideal environment for telomere recombination and DNA synthesis [19, 3236]. Indeed, disruption of APBs by depleting PML not only abolishes telomere clustering, but also blocks ALT telomeric DNA synthesis in G2 cells [37, 38]. However, how APBs are assembled and how they promote ALT activation is still not fully understood.

PML bodies are membraneless organelles assembled through multivalent SUMO-SIM (SUMO-interacting motif) interactions among PML molecules, a process with features of liquid-liquid phase separation (LLPS) $[39,40]$. A recent study proposed that APBs are also formed through LLPS (Fig. 1) [41]. In this study, fusion

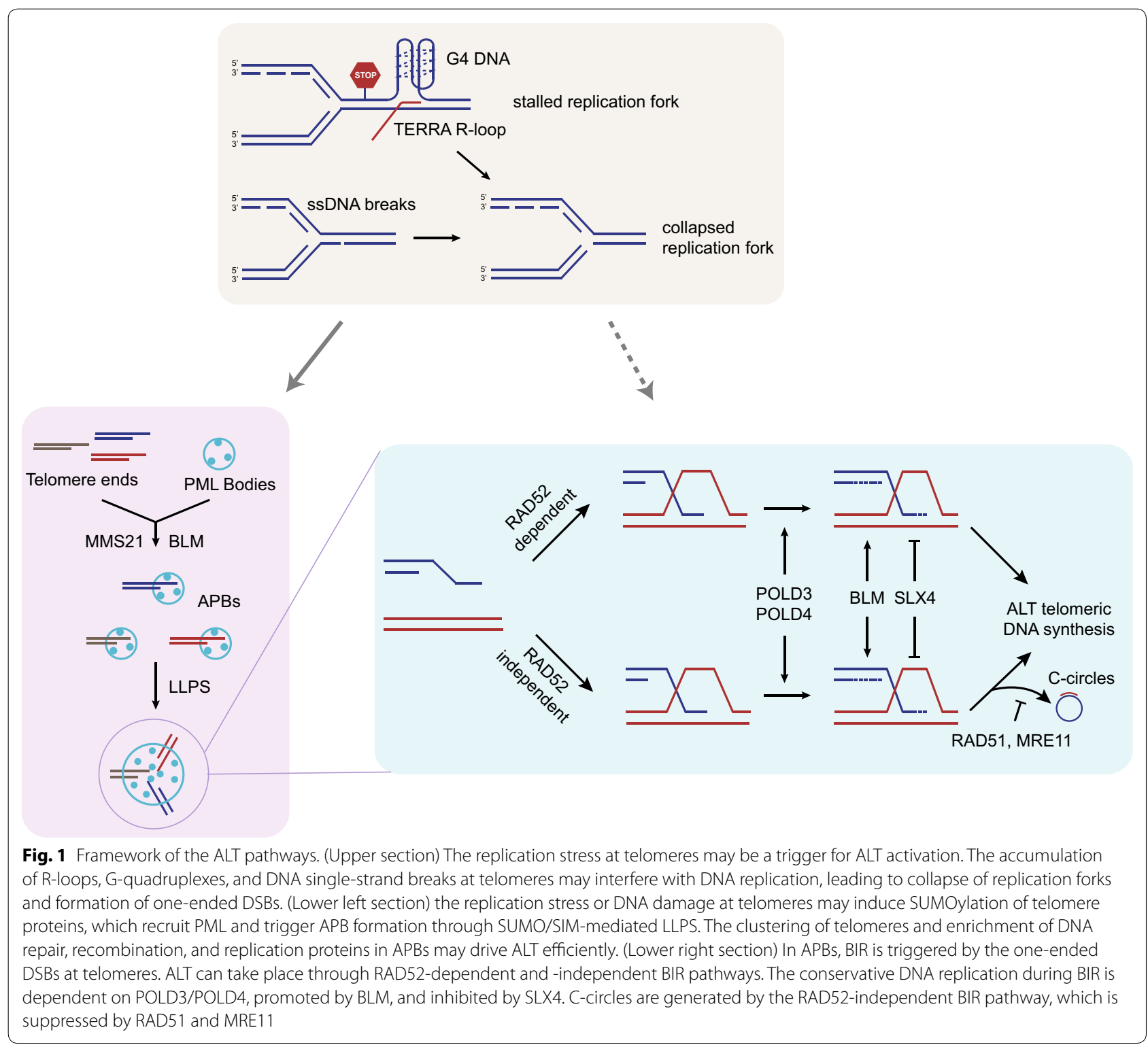


proteins containing multiple SUMO and SIMs were tethered to telomeres in $\mathrm{ALT}^{-}$cells, resulting in PML-telomere colocalization and telomere clustering in nuclear condensates resembling APBs in $\mathrm{ALT}^{+}$cells. Interestingly, although these poly-SUMO/SIM-induced condensates display features of LLPS, they are not sufficient for ALT DNA synthesis. Only when the BLM helicase was overexpressed in these ALT $^{-}$cells, several ALT-associated phenotypes, including heterogeneous telomere length, C-circles formation, and mitotic DNA synthesis (MiDAS) at telomeres, were detected [41]. It is possible that the enrichment of SUMO/SIM at telomeres recruits PML and triggers LLPS, driving multiple telomeres into condensates. However, clustering of telomeres does not appear to be sufficient to activate the ALT pathway. Overexpression of BLM apparently generates a signal critical for ALT activation in these telomere condensates. Interestingly, endogenous BLM is a component of APBs and critical for APB formation and ALT telomeric DNA synthesis $[35,38,42]$. While the induction of telomere LLPS and overexpression of BLM recapitulate important steps for ALT activation, how telomere LLPS and ALT activation are naturally triggered in $\mathrm{ALT}^{+}$cells remains to be investigated.

The formation of APBs has been linked to the replication stress or DNA damage at telomeres. Loss of proteins that suppress replication stress or DNA damage, such as SMARCAL1, FANCD2, and FANCM, increases APB formation [43-46]. The replication stress at telomeres may lead to collapse of replication forks, which may promote APB formation through MMS21-mediated SUMOylation of shelterin and other telomere-binding proteins [47]. Interestingly, overexpression of BLM or RAD52 promotes APB formation, telomere clustering, and MiDAS at telomeres [41, 48], whereas overexpression of SLX4 reduces APBs and shortens telomere extension [48]. These results suggest that BLM and RAD52 may play opposing roles from SLX4 in regulating ALT telomeric DNA synthesis, and that ALT telomeric DNA synthesis may also affect the dynamics of APBs.

\section{Framework of the ALT pathway}

The ALT pathway was initially characterized in the budding yeast mutant lacking functional telomerase [49]. In yeast, Rad51 is the key recombinase in the homologous recombination (HR) pathway [50]. Rad52 is critical for the binding of Rad51 to single-stranded DNA and also has the ability to anneal complementary ssDNA [51,52]. There are two distinct types of ALT in yeast: Rad51- and Rad52-dependent type I survivors maintain telomeres by amplifying repetitive subtelomeric sequences, and Rad52-dependent but Rad51-independent type II survivors maintain telomeres by expanding the telomeric repeats [53]. The extension of telomeres by the ALT pathway in human cells is thought to be more similar to that in type II survivors $[15,54]$. However, due to the lack of methods to directly monitor ALT DNA synthesis, little was known about how the ALT pathway operates in $\mathrm{ALT}^{+}$human cells. Recently, the development of several assays to model or monitor ALT DNA synthesis, including break-induced telomere synthesis (BITS) [55, 56], MiDAS at telomeres $[41,57,58]$, and ALT telomere synthesis in APBs (ATSA) [38], has allowed us to unravel the molecular mechanisms of ALT.

Break-induced replication (BIR) is a repair process initiated by one-ended DSBs at collapsed replication forks and extended by conservative DNA replication [59-61]. The yeast Pol32 and human POLD3/4 proteins, accessory subunits of the DNA polymerase, are important for BIR [56, 62, 63]. In $\mathrm{ALT}^{+}$human cells, telomeres undergo conservative DNA replication in a POLD3/4-dependent manner, thereby linking ALT to BIR [64]. Human RAD52 is important for BIR at collapsed forks and MiDAS at fragile sites $[65,66]$. Similar to that in the yeast type II survivors, the ALT pathway in human cells is dependent on RAD52 but not RAD51 [38, 41, 56, 57]. Telomeric DSBs generated by the TRF1-FOK1 nuclease fusion induce telomere clustering and BITS $[55,56]$. Depletion of RAD51 impairs telomere clustering but does not affect BITS. Perhaps because $\mathrm{ALT}^{+}$cells have high replication stress at telomeres, telomeric MiDAS is more robust in $\mathrm{ALT}^{+}$cells than in $\mathrm{ALT}^{-}$cells [57]. Telomeric MiDAS results in conservative DNA replication, recapitulating a phenotype of $\mathrm{ALT}^{+}$cells. Depletion or inhibition of RAD52 decreases telomeric MiDAS. In contrast, RAD51 depletion increases telomeric MiDAS, fragile telomeres, and telomere dysfunction-induced foci (TIF) [57]. We recently showed that depletion of RAD52 decreases ATSA, the natural ALT telomere synthesis at APBs in G2 cells [38]. Depletion of RAD51 has no effect on ATSA but increases C-circles, a marker of ALT [38, 67]. In vitro, RAD52 but not RAD51 promotes D-loop formation on telomeric DNA in the presence of RPA [38]. Overexpression of RAD52 promotes telomere synthesis in G2 and mitotic cells [41]. All these results suggest that RAD52 but not RAD51 is important for ALT in human cells. Instead of participating in ALT directly, RAD51 may suppress the fragility of telomeres by protecting stalled replication forks. However, considering that RAD51 was shown to be required for telomere extension in cells overexpressing BLM [48], we cannot exclude a role for RAD51 in a context-specific ALT pathway.

It is important to emphasize that the ALT pathway in human cells is not a simple linear pathway. In our recent studies, we noticed that although ATSA is decreased in the newly generated RAD52 KO cells, significant levels 
of ATSA remain detectable in the absence of RAD52 [38]. Furthermore, C-circles levels are not altered in RAD52 knockdown cells and newly generated RAD52 $\mathrm{KO}$ cells, suggesting that there is a RAD52-independent ALT pathway, which is responsible for C-circles generation. During the passage of RAD52 KO cells, telomeres are progressively shortened and eventually stabilized. Interestingly, C-circle levels are elevated in extensively passaged RAD52 KO cells, indicating that the RAD52independent pathway becomes more active when telomeres become shorter. Depletion of BLM, POLD3 and POLD4 decrease ATSA in extensively passaged RAD52 $\mathrm{KO}$ cells, showing that the RAD52-independent ALT pathway still depends on APBs and BIR for DNA synthesis. Based on these results, we proposed that ALT is a bifurcated pathway involving RAD52-dependent and RAD52-independent BIR, at least in G2 cells (Fig. 1) [38].

In human cells, the ALT pathway may alter according to the cell-cycle status of cells and the replication stress or DNA damage at telomeres. A low dose of aphidicolin induces RAD52-mediated telomeric MiDAS in not only $\mathrm{ALT}^{+}$but also $\mathrm{ALT}^{-}$cells $[57,58]$, suggesting that MiDAS is a general mechanism dealing with replication stress at telomeres. Depletion of SLX4 blocks telomeric MiDAS but increases APBs, C-circles and ALT telomere extension $[48,58]$. It is possible that telomeric MiDAS only represents a sub-pathway of ALT in M phase. In contrast to telomeric MiDAS, ATSA detects telomeric DNA synthesis at APBs in G2 cells without exogenous DNA damage or replication inhibitor [38]. ATSA is only observed in $\mathrm{ALT}^{+}$but not $\mathrm{ALT}^{-}$cells. Both RAD52-dependent and -independent ALT pathways contribute to ATSA in G2, but telomeric MiDAS seems to be largely if not entirely RAD52-dependent. Telomeric DSBs generated by TRF1FOK1 induce BITS in unsynchronized cells, with $\mathrm{ALT}^{+}$ cells displaying more DNA synthesis than $\mathrm{ALT}^{-}$cells [56]. Interestingly, both RAD51 and RAD52 are dispensable for BITS, suggesting that BITS may resemble the RAD52-independent ALT pathway [68]. Recently, RAD51AP1 was shown to be required for TRF1-FOK1induced telomere clustering and BITS [69]. Depletion of RAD51AP1 diminishes several ALT hallmarks, including APBs, T-SCE, ECTR, and the localization of RAD52 and POLD3 to telomeres. Moreover, knockout of RAD51AP1 leads to telomere shortening. In addition to DNA repair proteins, several nuclear receptors are also implicated in ALT. Recently, an ALT telomere maintenance pathway mediated by the COUP-TFII/TR4-FANCD2-MUS81POLD3 axis was reported [70]. The nuclear receptor NR2C/F may recruit the NuRD-ZNF827 complex to telomeres and promote telomere recombination through chromatin remodeling [23, 24, 71]. Further studies are needed to understand how these factors function in RAD52-dependent and/or -independent pathways.

\section{Regulatory circuitries of ALT}

The DNA replication stress at telomeres may be an important trigger of ALT activation (Fig. 1). The ATRXDAXX complex was shown to deposit the histone variant $\mathrm{H} 3.3$ to telomeric regions of the genome [72-74]. Mutations in ATRX, DAXX, and H3.3 are prevalent in $\mathrm{ALT}^{+}$cancers [26-28]. Expression of ATRX suppresses ALT markers including APBs and C-circles [75, 76], suggesting that ATRX is a repressor of ALT. Loss of ATRX results in dysregulation of the telomere non-coding RNA TERRA during the cell cycle and TERRA upregulation [77]. TERRA is known to form RNA:DNA hybrids at telomeres, inducing replication stress [78]. Loss of ATRX may also lead to accumulation of G-quadruplexes at ALT telomeres, which also impose replication stress [76, 79]. Some of the proteins bound to TERRA or RNA:DNA hybrids may act to reduce replication stress and suppress ALT. For example, TERRA-binding proteins SFPQ and NONO suppress telomeric RNA:DNA hybrids, and depletion of SFPQ increases APBs and telomere recombination in $\mathrm{ALT}^{+}$cells [80].

Both intrinsic and extrinsic DNA replication stress promote ALT-associated events [57, 81, 82], suggesting that the proteins involved in the replication stress response are important for ALT suppression. For example, the ATP-dependent DNA-annealing helicase SMARCAL1 may counter replication stress by promoting reversal of stalled replication forks [83]. SMARCAL1 associates with ALT telomeres and suppresses ALT phenotypes [84]. RAD51 is also required for fork reversal, and MRE11 is involved in the nucleolytic processing of revered replication forks [85-87]. Loss of RAD51 or MRE11 increases $\mathrm{C}$-circles, indicating that fork reversal and the subsequent processing may play an important role in suppressing C-circles formation [38, 81]. In addition, the fork protection complex (FPC) containing TIMELESS and TIPIN suppresses telomeric MiDAS [57].

Fanconi anemia (FA) proteins are also implicated in the suppression of ALT. FANCD2 suppresses BLM-dependent telomere extension in $\mathrm{ALT}^{+}$cells [44]. Recently, FANCM was also found to suppress ALT $[45,46,88]$. Loss of FANCM in $\mathrm{ALT}^{+}$cells enhances ALT phenotypes, including telomere clustering in APBs, C-circles formation, and telomeric DNA synthesis in G2 cells [45, 46]. In addition, depletion of FANCM leads to accumulation of phosphorylated RPA, single-stranded DNA, and foci of $\mathrm{H} 2 \mathrm{AX}$ and 53BP1 at ALT telomeres, showing that hyperactive ALT is associated with high levels of DNA damage at telomeres. Importantly, FANCM is found to be critical for the viability of $\mathrm{ALT}^{+}$cells but not $\mathrm{ALT}^{-}$cells $[45,46]$. 
The toxicity of FANCM depletion from $\mathrm{ALT}^{+}$cells may be attributed to several functions of FANCM in ALT suppression. The function of FANCM in ALT suppression is dependent its ATPase/translocase activity. FANCM restricts TERRA levels and suppresses telomeric R-loops, possibly through its R-loop unwinding activity [46]. In addition, FANCM interacts with BLM and may antagonize the function of BLM in ALT [46]. Finally, FANCM may suppress ALT by reversing and remodeling stalled replication forks to counter replication stress at telomeres, which is similar to the role of SMARCAL1 $[43,89]$

BLM and SLX4 appear to play opposing roles in ALT (Fig. 1). Overexpression of BLM induces telomere extension, APBs, and C-circles in $\mathrm{ALT}^{+}$cells, whereas depletion of BLM causes opposite effects [35, 48]. When APBs are induced in $\mathrm{ALT}^{-}$cells by tethering SUMO/ SIM fusions to telomeres, BLM overexpression triggers a number of ALT phenotypes [41]. Notably, the helicase activity of BLM is essential for ALT telomeric DNA synthesis [41]. In contrast to BLM, overexpression of SLX4 suppresses telomere extension, APBs, and C-circles [48]. It is proposed that SLX4 and its associated nucleases suppress ALT DNA synthesis by resolving intermediates of telomere extension. Recently, the SLX4-interacing protein SLX4IP was also shown to suppress ALT [90]. Interestingly, loss of BLM suppresses the exacerbated ALT phenotypes in cells lacking SLX4 and SLX4IP [90], which is reminiscent of the suppression of hyperactive ALT in FANCM-depleted cells by BLM loss [46]. In both cases, BLM activity seems to contribute to the hyper ALT phenotypes and the associated genomic instability.

Although BLM is clearly critical for ALT, its function in ALT is still an enigma. BLM might promote ALT by enhancing DSB end resection or processing/modeling recombination intermediates. In FANCM-depleted $\mathrm{ALT}^{+}$ cells, BLM may cause toxicity by driving hyper resection [46]. When APBs are induced in $\mathrm{ALT}^{-}$cells, BLM overexpression leads to accumulation of RPA at telomeres, consistent with a role of BLM in resection [41]. However, depletion of DNA2, the nuclease that functions with BLM in resection, fails to suppress ALT in cells lacking SLX4IP [90]. If BLM is important for processing/remodeling certain recombination intermediates during BIR, this function is likely antagonized by SLX4. The proper balance between BLM and SLX4 may be important for keeping ALT activity at a productive but tolerable level. While the hyperactivity of BLM may be a cause of ALTassociated genomic instability, it should be noted that BLM is required for APB formation and ALT DNA synthesis [38, 42]. Thus, loss of BLM is expected to block ALT and suppress the genomic instability associated with hyperactive ALT regardless of whether hyperactive BLM is the cause.
The Shelterin complex is critical for telomere maintenance regardless of the ALT status [91]. While some Shelterin proteins may be involved in ALT, it is generally difficult to separate their ALT functions from their roles in telomere protection. Nonetheless, some known functions of Shelterin proteins likely influence ALT activity. For example, loss of TRF1 increases telomere fragility, which may trigger ALT [92]. TRF2 is required for the induction of R-loops at telomeres after oxidative DNA damage, and it may also promote ALT by increasing telomeric R-loops [93]. Both TRF1 and TRF2 are SUMOylated by MMS21, allowing them to localize to APBs [94]. TRF1 may contribute to APB formation through its interaction with PMLIV [95]. Recently, it was shown that SUMOylation of TRF2 promotes telomere clustering, APB formation and Telomeric MiDAS [41].

\section{Therapeutic outlooks}

Although ALT is only active in about $4-11 \%$ of human cancers, it is prevalent in specific cancers, such as osteosarcoma, leiomyosarcoma, liposarcoma, glioblastoma, and neuroendocrine pancreatic cancer $[9,25,26]$. Moreover, the specific activation of ALT in tumors makes it a potential target for therapy. With the recent insights into the ALT pathway, several strategies have been proposed to exploit the dependency of tumors on ALT.

Since telomere length is heterogeneous in $\mathrm{ALT}^{+}$cells, inhibition of ALT may lead to shortening of already short telomeres, causing loss of telomeres, toxic chromosome fusions, and cell death. Because APBs are important for ALT DNA synthesis $[19,38]$, disrupting APBs may abolish ALT activity in $\mathrm{ALT}^{+}$cells. Indeed, disruption of APBs by PML knockdown results in telomere shortening in $\mathrm{ALT}^{+}$cells [37]. Similarly, knockdown of MMS21, a SUMO ligase required for APB formation, also causes telomere shortening in $\mathrm{ALT}^{+}$cells [47]. While blocking the ALT pathway may induce cell death in some $\mathrm{ALT}^{+}$ cells, whether this strategy can rapidly eliminate populations of $\mathrm{ALT}^{+}$cells in tumors is still unclear. $\mathrm{ALT}^{+}$cell populations seem to undergo progressive telomere shortening in the absence of ALT activity, and eventually enter senescence. Thus, blocking ALT in $\mathrm{ALT}^{+}$tumor cells may reduce their oncogenic potential, but may not eliminate them in a timely fashion.

A second strategy to kill $\mathrm{ALT}^{+}$cells is to induce ALTspecific synthetic lethality. ALT is a mechanism to extend short telomeres. If $\mathrm{ALT}^{+}$tumor cells accumulate high levels of DSBs at telomeres, they will be increasingly dependent on ALT activity to extend broken telomeres and survive. Inhibition of the ATR kinase, a master regulator of the replication stress response, increases telomere fragility [96, 97]. This effect of ATR inhibition may be attributed to both the increase of replication 


\section{Postive Regulators}

TERRA, G4-DNA

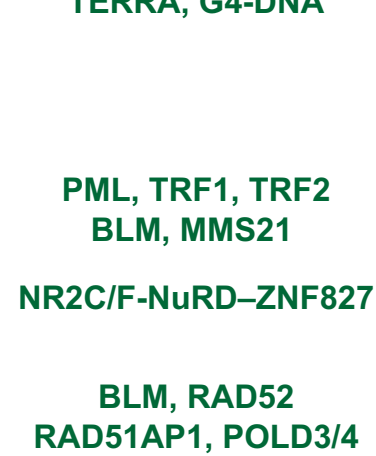

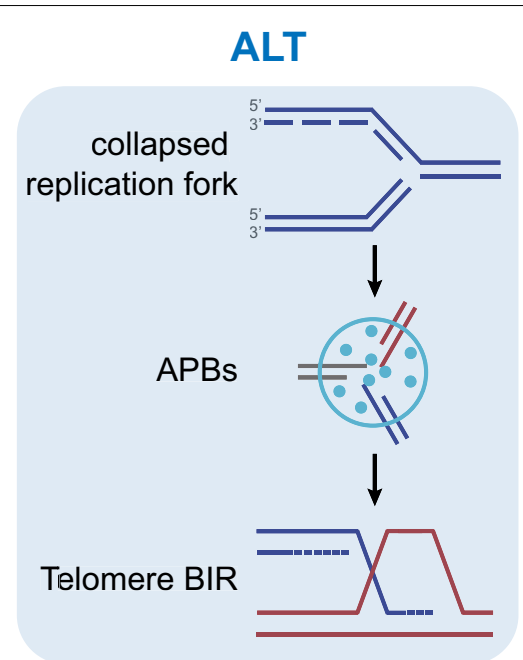

Negative Regulators

FANCD2, FANCM

SMARCAL1, RAD51

SLX4

SLX4, SLX4IP

Fig. 2 Positive and negative regulators of ALT. (Left) Positive regulators of ALT discussed in this article. (Middle) Major events that occur during the process of ALT. (Right) Negative regulators of ALT discussed in this article

stress at telomeres and the reduction in replication stress response. Furthermore, ATR is required for telomeric MiDAS in $\mathrm{ALT}^{+}$cells [57], suggesting that it may contribute to ALT activity. Thus, inhibition of ATR in $\mathrm{ALT}^{+}$cells not only induces DSBs at telomeres, but also prevents extension of broken telomeres through ALT, thereby creating a lethal situation in $\mathrm{ALT}^{+}$cells. Given the promising effects of ATR inhibitors on some $\mathrm{ALT}^{+}$cell lines [77], it would be important to test whether ATR inhibitors can effectively eliminate $\mathrm{ALT}^{+}$tumors in mouse models and patients.

As mentioned above, hyperactive ALT is associated with high levels of DNA damage at telomeres and may cause cell death. Therefore, strategies that induce hyperactive ALT may also selectively kill $\mathrm{ALT}^{+}$cells. For example, compounds that stabilize G-quadruplexes at telomeres may promote hyper activation of ALT, leading to death of $\mathrm{ALT}^{+}$cells [98]. Because loss of FANCM results in hyperactive ALT and death of $\mathrm{ALT}^{+}$cells, inhibitors of FANCM could potentially eliminate $\mathrm{ALT}^{+}$tumors [45, 46]. As a proof of principle, an ectopic MM2 peptide disrupting the interaction between FANCM and BLM is sufficient to decrease the survival of $\mathrm{ALT}^{+}$cells but has no effect on $\mathrm{ALT}^{-}$cells [45]. Similarly, the small molecule inhibitor PIP-199 disrupts the FANCM-BLM interaction and suppresses the growth of $\mathrm{ALT}^{+}$cancer cells.

Because ATRX is often lost in $\mathrm{ALT}^{+}$cells, the absence of ATRX in $\mathrm{ALT}^{+}$cells may provide a therapeutic opportunity. ATRX is involved in the restart of stalled replication forks [99], and in DNA repair synthesis and sister chromatid exchange during HR [100]. Loss of ATRX was shown to sensitize cells to DNA-damaging agents [101]. ATRX deficiency was also shown to promote accumulation of G-quadruplexes, making cells sensitive to a G4-stabilizing compound [102]. Furthermore, a mutant herpes simplex virus type 1 (HSV-1) infects ATRX-deficient cells much more efficiently than ATRX-proficient cells, selectively killing ATRX-deficient cells [103]. Thus, although strategies that selectively kill ATRX-deficient cells may not exploit the ALT pathway directly, they may eliminate the ATRX-deficient $\mathrm{ALT}^{+}$ tumors.

Targeting the ALT-specific telomere proteins may be another way to kill $\mathrm{ALT}^{+}$cells. TSPYL5 is a protein that is specifically expressed in $\mathrm{ALT}^{+}$cancer cells [104]. Interestingly, depletion of TSPYL5 induces USP7dependent proteasomal degradation of POT1, a component of the shelterin complex, leading to death of $\mathrm{ALT}^{+}$cells. Furthermore, knockdown of PML prevents POT1 degradation in $\mathrm{ALT}^{+}$cells lacking TSPYL5, suggesting that POT1 becomes reliant on TSPYL5 in APBs. This finding provides an example of how ALT and APB can change the behaviors of telomere binding proteins, suggesting that targeting ALT-specific telomere proteins like TSPYL5 may induce genomic instability specifically at ALT telomeres, killing $\mathrm{ALT}^{+}$cells selectively.

\section{Conclusions}

In this review, we attempted to summarize the recent studies on the molecular basis of the ALT pathways. Collectively, these studies suggest that ALT is a complex BIR pathway initiated by the replication stress or DNA damage at telomeres. Although several key events for ALT activation are uncovered, how this pathway is specifically activated in $\mathrm{ALT}^{+}$cells still requires further 
investigations. ALT activity can be detected at APBs in G2 cells, and even in mitotic cells. A number of positive and negative regulators of ALT have been identified (Fig. 2), but how they function together is still not fully understood. It is important to note that many of the current approaches to study ALT still rely on exogenous DNA damage or replication inhibitors. How the natural ALT activity in $\mathrm{ALT}^{+}$cells is regulated remains an outstanding question. With a better understanding of the ALT pathway, we expect that more therapeutic strategies will be developed to exploit the ALT-associated vulnerabilities of $\mathrm{ALT}^{+}$tumors. The next decade will be an exciting time for both basic and translational research of ALT.

\section{Abbreviations}

ALT: Alternative lengthening of telomeres; APBs: ALT-associated PML bodies; ATSA: ALT telomere synthesis in APBs; BIR: Break-induced replication; BITS: Break-induced telomere synthesis; DSBs: DNA double-strand breaks; ECTR: Extrachromosomal telomere repeat; HR: Homologous recombination; LLPS: Liquid-liquid phase separation; MiDAS: Mitotic DNA synthesis; PML: Promyelocytic leukemia; T-SCE: Telomere sister chromatid exchange.

\section{Acknowledgements}

We thank the members of Zou, Lan, and Dyson laboratories for discussions.

\section{Authors' contributions}

J-MZ and LZ wrote the paper. Both authors read and approved the final manuscript.

\section{Funding}

J.-M. Z. is a recipient of the MGH Cancer Center Excellence Award. L. Z. is the James and Patricia Poitras Endowed Chair for Cancer Research.

\section{Ethical approval and consent to participate.}

Not applicable.

\section{Consent for publication}

Not applicable.

\section{Competing interests}

The authors declares that they have no competing interests.

\section{Author details \\ ${ }^{1}$ Massachusetts General Hospital Cancer Center, Harvard Medical School, Charlestown, MA 02129, USA. ${ }^{2}$ Department of Pathology, Massachusetts General Hospital, Harvard Medical School, Boston, MA 02114, USA.}

Received: 8 December 2019 Accepted: 23 February 2020

Published online: 10 March 2020

\section{References}

1. Palm W, de Lange T. How shelterin protects mammalian telomeres. Annu Rev Genet. 2008;42:301-34.

2. O'Sullivan RJ, Karlseder J. Telomeres: protecting chromosomes against genome instability. Nat Rev Mol Cell Biol. 2010;11(3):171-81.

3. Shay JW, Wright WE. Telomeres and telomerase: three decades of progress. Nat Rev Genet. 2019;20(5):299-309.

4. Bernal A, Tusell L. Telomeres: implications for cancer development. Int J Mol Sci. 2018;19(1):294.

5. Hanahan D, Weinberg RA. Hallmarks of cancer: the next generation. Cell. 2011;144(5):646-74.
6. Kim NW, et al. Specific association of human telomerase activity with immortal cells and cancer. Science. 1994;266(5193):2011-5.

7. Shay JW, Bacchetti S. A survey of telomerase activity in human cancer Eur J Cancer. 1997;33(5):787-91.

8. Barthel FP, et al. Systematic analysis of telomere length and somatic alterations in 31 cancer types. Nat Genet. 2017:49(3):349-57.

9. Heaphy $\mathrm{CM}$, et al. Prevalence of the alternative lengthening of telomeres telomere maintenance mechanism in human cancer subtypes. Am J Pathol. 2011:179(4):1608-15.

10. Dilley RL, Greenberg RA. Alternative telomere maintenance and cancer. Trends Cancer. 2015;1(2):145-56

11. Cesare AJ, Reddel RR. Alternative lengthening of telomeres: models, mechanisms and implications. Nat Rev Genet. 2010;11(5):319-30.

12. Sobinoff AP, Pickett HA. Alternative lengthening of telomeres: DNA repair pathways converge. Trends Genet. 2017;33(12):921-32.

13. De Vitis M, Berardinelli F, Sgura A. Telomere length maintenance in cancer: at the crossroad between telomerase and alternative lengthening of telomeres (ALT). Int J Mol Sci. 2018;19(2):606

14. Lundblad V, Blackburn EH. An alternative pathway for yeast telomere maintenance rescues est1- senescence. Cell. 1993;73(2):347-60.

15. Bryan $\mathrm{TM}$, et al. Telomere elongation in immortal human cells without detectable telomerase activity. EMBO J. 1995;14(17):4240-8.

16. Bryan $\mathrm{TM}$, et al. Evidence for an alternative mechanism for maintaining telomere length in human tumors and tumor-derived cell lines. Nat Med. 1997:3(11):1271-4.

17. Dunham MA, et al. Telomere maintenance by recombination in human cells. Nat Genet. 2000;26(4):447-50.

18. Muntoni A, et al. Telomere elongation involves intra-molecular DNA replication in cells utilizing alternative lengthening of telomeres. Hum Mol Genet. 2009;18(6):1017-27.

19. Yeager TR, et al. Telomerase-negative immortalized human cells contain a novel type of promyelocytic leukemia (PML) body. Cancer Res. 1999:59(17):4175-9.

20. Cesare AJ, Griffith JD. Telomeric DNA in ALT cells is characterized by free telomeric circles and heterogeneous t-loops. Mol Cell Biol. 2004;24(22):9948-57.

21. Tokutake $\mathrm{Y}$, et al. Extra-chromosomal telomere repeat DNA in telomerase-negative immortalized cell lines. Biochem Biophys Res Commun. 1998;247(3):765-72

22. Londono-Vallejo JA, et al. Alternative lengthening of telomeres is characterized by high rates of telomeric exchange. Cancer Res. 2004:64(7):2324-7.

23. Conomos D, et al. Variant repeats are interspersed throughout the telomeres and recruit nuclear receptors in ALT cells. J Cell Biol. 2012;199(6):893-906.

24. Marzec $P$, et al. Nuclear-receptor-mediated telomere insertion leads to genome instability in ALT cancers. Cell. 2015;160(5):913-27.

25. Henson JD, Reddel RR. Assaying and investigating alternative lengthening of telomeres activity in human cells and cancers. FEBS Lett. 2010;584(17):3800-11

26. Heaphy CM, et al. Altered telomeres in tumors with ATRX and DAXX mutations. Science. 2011;333(6041):425.

27. Schwartzentruber J et al. Driver mutations in histone $\mathrm{H} 3.3$ and chromatin remodelling genes in paediatric glioblastoma. Nature. 2012:482(7384):226-31.

28. Lovejoy CA, et al. Loss of ATRX, genome instability, and an altered DNA damage response are hallmarks of the alternative lengthening of telomeres pathway. PLoS Genet. 2012;8(7):e1002772.

29. Dyer MA, et al. ATRX and DAXX: mechanisms and mutations. Cold Spring Harb Perspect Med. 2017;7(3):a026567.

30. Jiao $Y$, et al. DAXX/ATRX, MEN1, and mTOR pathway genes are frequently altered in pancreatic neuroendocrine tumors. Science. 2011;331(6021):1199-203.

31. Kannan $\mathrm{K}$, et al. Whole-exome sequencing identifies ATRX mutation as a key molecular determinant in lower-grade glioma. Oncotarget. 2012:3(10):1194-203.

32. Grobelny JV, Godwin AK, Broccoli D. ALT-associated PML bodies are present in viable cells and are enriched in cells in the $\mathrm{G}(2) / \mathrm{M}$ phase of the cell cycle. J Cell Sci. 2000;1 13(Pt 24):4577-85.

33. Wu G, Lee WH, Chen PL. NBS1 and TRF1 colocalize at promyelocytic leukemia bodies during late S/G2 phases in immortalized 
telomerase-negative cells. Implication of NBS1 in alternative lengthening of telomeres. J Biol Chem. 2000;275(39):30618-22.

34. Draskovic l, et al. Probing PML body function in ALT cells reveals spatiotemporal requirements for telomere recombination. Proc Natl Acad Sci U S A. 2009;106(37):15726-31.

35. Stavropoulos DJ, et al. The Bloom syndrome helicase BLM interacts with TRF2 in ALT cells and promotes telomeric DNA synthesis. Hum Mol Genet. 2002;11(25):3135-44.

36. Nabetani A, Yokoyama O, Ishikawa F. Localization of hRad9, hHus1, hRad1, and hRad17 and caffeine-sensitive DNA replication at the alternative lengthening of telomeres-associated promyelocytic leukemia body. J Biol Chem. 2004;279(24):25849-57.

37. Osterwald $\mathrm{S}$, et al. PML induces compaction, TRF2 depletion and DNA damage signaling at telomeres and promotes their alternative lengthening. J Cell Sci. 2015;128(10):1887-900.

38. Zhang $\mathrm{JM}$, et al. Alternative lengthening of telomeres through two distinct break-induced replication pathways. Cell Rep. 2019;26(4):955-68.

39. Shen TH, et al. The mechanisms of PML-nuclear body formation. Mol Cell. 2006;24(3):331-9.

40. Banani SF, et al. Compositional control of phase-separated cellular bodies. Cell. 2016;166(3):651-63.

41. Min J, Wright WE, Shay JW. Clustered telomeres in phase-separated nuclear condensates engage mitotic DNA synthesis through BLM and RAD52. Genes Dev. 2019;33(13-14):814-27.

42. O'Sullivan RJ, et al. Rapid induction of alternative lengthening of telomeres by depletion of the histone chaperone ASF1. Nat Struct Mol Biol. 2014;21(2):167-74.

43. Cox KE, Marechal A, Flynn RL. SMARCAL1 resolves replication stress at ALT telomeres. Cell Rep. 2016;14(5):1032-40.

44. Root $\mathrm{H}$, et al. FANCD2 limits BLM-dependent telomere instability in the alternative lengthening of telomeres pathway. Hum Mol Genet. 2016;25(15):3255-68.

45. Lu R, et al. The FANCM-BLM-TOP3A-RMI complex suppresses alternative lengthening of telomeres (ALT). Nat Commun. 2019;10(1):2252.

46. Silva $B$, et al. FANCM limits ALT activity by restricting telomeric replication stress induced by deregulated BLM and R-loops. Nat Commun. 2019;10(1):2253.

47. Potts PR, Yu H. The SMC5/6 complex maintains telomere length in ALT cancer cells through SUMOylation of telomere-binding proteins. Nat Struct Mol Biol. 2007;14(7):581-90.

48. Sobinoff AP, et al. BLM and SLX4 play opposing roles in recombination-dependent replication at human telomeres. EMBO J. 2017;36(19):2907-19.

49. McEachern MJ, Haber JE. Break-induced replication and recombinational telomere elongation in yeast. Annu Rev Biochem. 2006;75:111-35.

50. Krogh BO, Symington LS. Recombination proteins in yeast. Annu Rev Genet. 2004;38:233-71.

51. Mortensen $\mathrm{UH}$, et al. DNA strand annealing is promoted by the yeast Rad52 protein. Proc Natl Acad Sci U S A. 1996;93(20):10729-34.

52. New JH, et al. Rad52 protein stimulates DNA strand exchange by Rad51 and replication protein A. Nature. 1998;391 (6665):407-10.

53. Le S, et al. RAD50 and RAD51 define two pathways that collaborate to maintain telomeres in the absence of telomerase. Genetics. 1999;152(1):143-52.

54. Teng SC, Zakian VA. Telomere-telomere recombination is an efficient bypass pathway for telomere maintenance in Saccharomyces cerevisiae. Mol Cell Biol. 1999;19(12):8083-93.

55. Cho NW, et al. Interchromosomal homology searches drive directional ALT telomere movement and synapsis. Cell. 2014;159(1):108-21.

56. Dilley RL, et al. Break-induced telomere synthesis underlies alternative telomere maintenance. Nature. 2016;539(7627):54-8.

57. Min J, Wright WE, Shay JW. Alternative lengthening of telomeres mediated by mitotic DNA synthesis engages break-induced replication processes. Mol Cell Biol. 2017;37(20):e00226-17.

58. Ozer $\mathrm{O}$, et al. Human cancer cells utilize mitotic DNA synthesis to resist replication stress at telomeres regardless of their telomere maintenance mechanism. Oncotarget. 2018;9(22):15836-46.

59. Anand RP, Lovett ST, Haber JE. Break-induced DNA replication. Cold Spring Harb Perspect Biol. 2013;5(12):a010397.
60. Malkova A, Ira G. Break-induced replication: functions and molecular mechanism. Curr Opin Genet Dev. 2013;23(3):271-9.

61. Kramara J, Osia B, Malkova A. Break-induced replication: the where, the why, and the how. Trends Genet. 2018;34(7):518-31.

62. Lydeard JR, et al. Break-induced replication and telomerase-independent telomere maintenance require Pol32. Nature. 2007;448(7155):820-3.

63. Costantino $L$, et al. Break-induced replication repair of damaged forks induces genomic duplications in human cells. Science. 2014;343(6166):88-91.

64. Roumelioti FM, et al. Alternative lengthening of human telomeres is a conservative DNA replication process with features of break-induced replication. EMBO Rep. 2016;17(12):1731-7.

65. Bhowmick R, Minocherhomji S, Hickson ID. RAD52 Facilitates mitotic dna synthesis following replication stress. Mol Cell. 2016;64(6):1117-26.

66. Sotiriou SK, et al. Mammalian RAD52 functions in break-induced replication repair of collapsed DNA replication forks. Mol Cell. 2016;64(6):1127-34.

67. Henson JD, et al. DNA C-circles are specific and quantifiable markers of alternative-lengthening-of-telomeres activity. Nat Biotechnol. 2009;27(12):1181-5.

68. Verma P, et al. RAD52 and SLX4 act nonepistatically to ensure telomere stability during alternative telomere lengthening. Genes Dev. 2019;33(3-4):221-35.

69. Barroso-Gonzalez J, et al. RAD51AP1 is an essential mediator of alternative lengthening of telomeres. Mol Cell. 2019;76(1):217.

70. Xu M, et al. Nuclear receptors regulate alternative lengthening of telomeres through a novel noncanonical FANCD2 pathway. Sci Adv. 2019;5(10):eaax6366.

71. Conomos D, Reddel RR, Pickett HA. NuRD-ZNF827 recruitment to telomeres creates a molecular scaffold for homologous recombination. Nat Struct Mol Biol. 2014;21(9):760-70.

72. Goldberg AD, et al. Distinct factors control histone variant H3.3 localization at specific genomic regions. Cell. 2010;140(5):678-91.

73. Lewis PW, et al. Daxx is an H3.3-specific histone chaperone and cooperates with ATRX in replication-independent chromatin assembly at telomeres. Proc Natl Acad Sci U S A. 2010;107(32):14075-80.

74. Wong $L H$, et al. ATRX interacts with $\mathrm{H} 3.3$ in maintaining telomere structural integrity in pluripotent embryonic stem cells. Genome Res. 2010;20(3):351-60.

75. Napier $C E$, et al. ATRX represses alternative lengthening of telomeres. Oncotarget. 2015;6(18):16543-58.

76. Clynes $D$, et al. Suppression of the alternative lengthening of telomere pathway by the chromatin remodelling factor ATRX. Nat Commun. 2015:6:7538.

77. Flynn RL, et al. Alternative lengthening of telomeres renders cancer cells hypersensitive to ATR inhibitors. Science. 2015;347(6219):273-7.

78. Arora R, et al. RNaseH1 regulates TERRA-telomeric DNA hybrids and telomere maintenance in ALT tumour cells. Nat Commun. 2014;5:5220.

79. Law MJ, et al. ATR-X syndrome protein targets tandem repeats and influences allele-specific expression in a size-dependent manner. Cell. 2010;143(3):367-78.

80. Petti $\mathrm{E}$, et al. SFPQ and NONO suppress RNA:DNA-hybrid-related telomere instability. Nat Commun. 2019;10(1):1001.

81. Zhang T, et al. Strand break-induced replication fork collapse leads to C-circles, C-overhangs and telomeric recombination. PLoS Genet. 2019;15(2):e1007925.

82. Cesare AJ, et al. Spontaneous occurrence of telomeric DNA damage response in the absence of chromosome fusions. Nat Struct Mol Biol. 2009;16(12):1244-51.

83. Betous R, et al. SMARCAL1 catalyzes fork regression and holliday junction migration to maintain genome stability during DNA replication. Genes Dev. 2012;26(2):151-62.

84. Poole LA, et al. SMARCAL1 maintains telomere integrity during DNA replication. Proc Natl Acad Sci U S A. 2015;112(48):14864-9.

85. Schlacher $\mathrm{K}$, et al. Double-strand break repair-independent role for BRCA2 in blocking stalled replication fork degradation by MRE11. Cell. 2011;145(4):529-42.

86. Zellweger $\mathrm{R}$, et al. Rad51-mediated replication fork reversal is a global response to genotoxic treatments in human cells. J Cell Biol. 2015;208(5):563-79. 
87. Hashimoto Y, et al. Rad51 protects nascent DNA from Mre11-dependent degradation and promotes continuous DNA synthesis. Nat Struct Mol Biol. 2010;17(11):1305-11.

88. Pan $\mathrm{X}$, et al. FANCM, BRCA1, and BLM cooperatively resolve the replication stress at the ALT telomeres. Proc Natl Acad Sci U S A. 2017;114(29):E5940-E59495949.

89. Gari K, et al. Remodeling of DNA replication structures by the branch point translocase FANCM. Proc Natl Acad Sci U S A. 2008:105(42):16107-12.

90. Panier S, et al. SLX4IP antagonizes promiscuous BLM activity during ALT maintenance. Mol Cell. 2019;76(1):27-43 e11.

91. de Lange T. Shelterin-mediated telomere protection. Annu Rev Genet. 2018;52:223-47.

92. Sfeir A, et al. Mammalian telomeres resemble fragile sites and require TRF1 for efficient replication. Cell. 2009;138(1):90-103.

93. Tan J, et al. An R-loop-initiated CSB-RAD52-POLD3 pathway suppresses ROS-induced telomeric DNA breaks. Nucleic Acids Res. 2020;48(3):1285-300.

94. Jiang WQ, et al. Identification of candidate alternative lengthening of telomeres genes by methionine restriction and RNA interference. Oncogene. 2007;26(32):4635-47.

95. Yu J, et al. PML3 interacts with TRF1 and is essential for ALT-associated PML bodies assembly in U2OS cells. Cancer Lett. 2010;291(2):177-86.

96. McNees CJ, et al. ATR suppresses telomere fragility and recombination but is dispensable for elongation of short telomeres by telomerase. J Cell Biol. 2010;188(5):639-52.
97. Pennarun $\mathrm{G}$, et al. ATR contributes to telomere maintenance in human cells. Nucleic Acids Res. 2010;38(9):2955-63.

98. Asamitsu S, et al. Recent progress of targeted g-quadruplex-preferred ligands toward cancer therapy. Molecules. 2019;24(3):429.

99. Leung JW, et al. Alpha thalassemia/mental retardation syndrome X-linked gene product ATRX is required for proper replication restart and cellular resistance to replication stress. J Biol Chem. 2013;288(9):6342-50.

100. Juhasz S, et al. ATRX promotes DNA repair synthesis and sister chromatid exchange during homologous recombination. Mol Cell. 2018;71(1):11-24 e7.

101. Conte D, et al. Loss of Atrx sensitizes cells to DNA damaging agents through p53-mediated death pathways. PLoS ONE. 2012;7(12):e52167.

102. Wang Y, et al. G-quadruplex DNA drives genomic instability and represents a targetable molecular abnormality in ATRX-deficient malignant glioma. Nat Commun. 2019;10(1):943.

103. Han M, et al. Synthetic lethality of cytolytic HSV-1 in cancer cells with ATRX and PML deficiency. J Cell Sci. 2019;132(5):jcs222349.

104. Episkopou $\mathrm{H}$, et al. TSPYL5 depletion induces specific death of ALT cells through USP7-dependent proteasomal degradation of POT1. Mol Cell. 2019;75(3):469-482 e6.

\section{Publisher's Note}

Springer Nature remains neutral with regard to jurisdictional claims in published maps and institutional affiliations.
Ready to submit your research? Choose BMC and benefit from:

- fast, convenient online submission

- thorough peer review by experienced researchers in your field

- rapid publication on acceptance

- support for research data, including large and complex data types

- gold Open Access which fosters wider collaboration and increased citations

- maximum visibility for your research: over $100 \mathrm{M}$ website views per year

At BMC, research is always in progress.

Learn more biomedcentral.com/submissions 